\title{
Eficacia de la radiofrecuencia convencional de geniculados para el tratamiento del dolor en gonartrosis moderada-severa
}

\author{
I. Ramírez Ogalla, A. Moreno Martín, M.M. Santana Pineda y F. Rodríguez Huertas \\ Servicio de Anestesia. Hospital de Jerez de la Frontera, Cádiz
}

Ramírez Ogalla I, Moreno Martín A, Santana Pineda MM, Rodríguez Huertas F. Eficacia de la radiofrecuencia convencional de geniculados para el tratamiento del dolor en gonartrosis moderada-severa. Rev Soc Esp Dolor 2014; 21(4): 212-218.

\begin{abstract}
Introduction: Knee osteoarthritis is a common joint disease in the elderly characterized by pain, stiffness and functional limitation, and daily life disability.

Objective: The purpose of this study was to assess the outcome in terms of pain, functional recovery, and side effects during six month of radiofrequency treatment applied to the articular nerve branches (genicular nerves) in patients with chronic knee pain. As secondary outcome we investigated pharmacological therapy reduction and patient's satisfaction after treatment.

Material and methods: This prospective, uncontrolled study involved 16 elderly patients with severe knee osteoarthritis pain after genicular nerve radiofrequency treatment. Visual analogic scale (VAS) was measured at baseline (before treatment), at one, three and six months post-procedure. For measurement of functional limitation we used the Western Ontario and McMaster Universities Osteoarthritis Index (WOMAC) before treatment, and three and six months after procedure. Treatment response was defined as at least $50 \%$ of VAS reduction. We also registered patient's satisfaction after treatment.

Results: We included 16 patients with severe knee osteoarthritis pain lasting more than three months, VAS score higher than 5, severe osteoarthritis in Kellgren-Lawrence classification (3-4) and pain resistant to conventional care. We found significant differences in the mean VAS value before radiofre-
\end{abstract}

Recibido: 15-07-13.

Aceptado: 25-10-13 quency 7 (6-8) and after one 3,5 (1-6), three 3,3 (1-7) and six months $3,37(1-8)$ after treatment. $75 \%$ of patients had $50 \%$ of VAS score reduction after one month, $68,75 \%$ of patients after three months and $65 \%$ of patients after six months ( $p=0,009)$. Moreover, we also found significant differences in WOMAC score mean values before treatment 65,8 (54-79) and after three 54,7 (41-67) and six 50,3 (37-69) months after radiofrequency. No patient reported a post-procedure adverse event during de follow-up period.

Conclusions: RF application to genicular nerves showed significant benefit in terms of pain reduction and functional improvement in a 6 month follow-up in patients with chronic knee osteoarthritis and thus may be an effective treatment in such cases. Further trials with larger sample size and longer follow-up are needed.

Key words: Radiofrequency. Knee. Osteoarthritis. Chronic pain. Genicular nerve.

\section{RESUMEN}

Introducción: la artrosis de rodilla o gonartrosis es una de las patologías articulares más comunes y extendidas en la edad avanzada, que se caracteriza, entre otros, por ocasionar dolor, rigidez e incapacidad funcional en un gran número de casos dificultando las actividades de la vida diaria.

Objetivo: nuestro objetivo consistió en evaluar la eficacia del tratamiento con radiofrecuencia convencional de nervios geniculados en pacientes con gonartrosis rebelde a medidas conservadoras, en relación al dolor y mejoría de la rigidez y mejorar la funcionalidad, así como registrar los efectos adversos de la técnica en la gonartrosis. Como objetivo secundario nos planteamos valorar la reducción en el consumo de fármacos antiálgicos, así como la satisfacción de los pacientes tras esta nueva posibilidad terapéutica.

Material y métodos: estudio prospectivo no controlado a una serie de pacientes con dolor moderado a severo por artrosis de rodilla a los que se realizó radiofrecuencia convencional de nervios geniculados de la rodilla. La medición del dolor se 
realizó mediante escala visual analógica (EVA) basal, al mes, a los 3 y a los 6 meses, y para la evaluación de la función se aplicó el cuestionario de Ontario McMaster Universidades Western (WOMAC) a los 3 y 6 meses tras el tratamiento. La respuesta al tratamiento se definió como $\geq 50 \%$ de disminución de la EVA del dolor. Así mismo se registró el grado de satisfacción de los pacientes con la técnica.

Resultados: se incluyeron 16 pacientes remitidos a nuestra Unidad con el diagnóstico de gonartrosis con dolor de más 3 meses de evolución, una intensidad del dolor medida mediante la escala VAS $\geq 5$ y escala radiológica de severidad de Kellgren-Lawrence 3-4, en los que habían fracasado tratamientos anteriores. Se encontraron diferencias estadísticamente significativas en el valor del EVA inicial 7 (6-8) y el valor al mes 3,5 (1-6), tres 3,3 (1-7) y seis meses 3,37 (1-8) ( $p=0,009)$. El $75 \%$ de los pacientes presentó una reducción del EVA $\geq 50 \%$ en el primer mes, el 68,7 \% a los 3 meses, y el $65 \%$ a los 6 meses de tratamiento. Con respecto a la mejoría del dolor y capacidad funcional medida mediante la escala WOMAC, se obtuvo una media inicial de 65,8 (54-79), de 54,7 (41-67) a los tres meses y 50,3 (37-69) a los seis meses, siendo estas diferencias estadísticamente significativas. En ningún caso hubo un empeoramiento de la sintomatología de los pacientes. No se objetivaron complicaciones serias derivadas de la técnica.

Conclusiones: en conclusión, la RF convencional parece reducir de manera significativa la intensidad del dolor en un periodo de 6 meses en más del $50 \%$ de los pacientes, al igual que mejora la capacidad funcional en pacientes diagnosticados de artrosis de rodilla. Son necesarios estudios más amplios, comparativos y prospectivos para poder realizar la evaluación de la eficacia de esta técnica.

Palabras clave: Radiofrecuencia. Rodilla. Gonartrosis. Dolor crónico. Nervios geniculados.

\section{INTRODUCCIÓN}

La artrosis de rodilla o gonartrosis es una de las patologías articulares más comunes y extendidas en la edad avanzada, que se caracteriza entre otros, por ocasionar dolor, rigidez e incapacidad funcional en un gran número de casos dificultando las actividades de la vida diaria (1).

Su incidencia aumenta con la edad y debido al envejecimiento de la población, su prevalencia está aumentando; según el estudio EPISER en nuestro país se estima una prevalencia de artrosis sintomática de rodilla del $10,2 \%$, $14 \%$ en mujeres y $5,7 \%$ en hombres. Existe evidencia radiográfica de artrosis en más del $60 \%$ de las personas alrededor de los 65 años, y en más del $80 \%$ de los mayores de $75(2,3)$.

Los factores que mostraron una asociación significativa con el riesgo de padecer artrosis de rodilla fueron: el sexo femenino, la edad mayor de 50 años y la obesidad (1).

Aunque la mayoría de los pacientes en estadios leves o dolor moderado responden a los tratamientos conservado- res, tales como el reposo relativo, la terapia física y el abordaje farmacológico, pero cuando esto falla, o bien cuando los efectos secundarios son intolerables, se debe iniciar el tratamiento intervencionista o la cirugía $(4,5)$. Sin embargo, hay determinados pacientes que por las comorbilidades que presentan son desestimados para reemplazo articular, y otros que no desean someterse a la intervención, o están en lista de espera quirúrgica. Existe un consenso en cuanto a la importancia del manejo individualizado e integral en el paciente con gonartrosis. Como el número de este tipo de pacientes irá incrementándose con el envejecimiento de la población, son necesarios abordajes alternativos a los tratamientos convencionales para el tratamiento del dolor de la artrosis (6).

La radiofrecuencia (RF) es una técnica intervencionista mínimamente invasiva que ofrece una alternativa más en el manejo del dolor crónico y está siendo cada vez más utilizada en los últimos años en las Unidades de Dolor Crónico. Existen ya algunas publicaciones que avalan la eficacia de la radiofrecuencia en el tratamiento de dolor radicular, síndrome facetario, sacroileítis, neuralgia del trigémino (7). Las ramas nerviosas que rodean la articulación de la rodilla se denominan nervios geniculados, son exclusivamente sensitivos y pueden ser localizados fácilmente de forma percutánea guiados por fluoroscopia y un neuroestimulador sensitivo-motor (8). Así pues, en pacientes con dolor moderado-severo por gonartrosis, el tratamiento con radiofrecuencia convencional sobre los nervios geniculados que rodean la cápsula articular de la rodilla podría ser una alternativa eficaz, con pocas complicaciones.

Ante la escasa evidencia científica publicada al respecto, nos planteamos evaluar la eficacia del tratamiento con radiofrecuencia convencional de nervios geniculados en pacientes con gonartrosis rebelde a medidas conservadoras, en relación al dolor y mejoría de la rigidez y mejorar la funcionalidad así como registrar los efectos adversos de la técnica en la gonartrosis. Como objetivo secundario nos planteamos valorar tanto la reducción en el consumo de fármacos antiálgicos, como la satisfacción de los pacientes tras esta nueva opción terapéutica.

\section{METODOLOGÍA}

Se trata de un estudio observacional prospectivo, longitudinal, no controlado realizado en la Unidad de Dolor del Servicio de Anestesia del Hospital SAS de Jerez durante 1 año. Se incluyeron pacientes (mayores de 65 años) remitidos a nuestra Unidad con el diagnóstico de gonartrosis con dolor de más 3 meses de evolución, una intensidad del dolor medida mediante la escala VAS $\geq 5$ y escala radiológica de severidad de Kellgren-Lawrence 3-4, en los que habían fracasado tratamientos anteriores (terapia farmacológica, rehabilitación e infiltraciones intraarticulares), en 
lista de espera quirúrgica o en aquellos en los que se había desestimado la cirugía protésica por patología concomitante. Se les programaba para tratamiento con radiofrecuencia convencional de nervios geniculados a nivel de rodilla con el objetivo de aliviar el dolor y mejorar la funcionalidad y su calidad de vida. A todos los pacientes se les explicó detalladamente en qué consistía el tratamiento, así como los posibles riesgos o complicaciones, formalizando todos los pacientes el consentimiento informado previo a su incorporación al estudio. Así mismo se les informó que en cualquier momento podían solicitar la no participación en el estudio, sin consecuencia alguna. Los criterios de exclusión aplicados fueron pacientes con alteraciones de la coagulación y anticoagulados, portadores de marcapasos, patología psiquiátrica, pacientes con discapacidad mental que impida la comunicación o colaboración, y aquellos pacientes que recibieran alguna infiltración articular de la rodilla tres meses antes del procedimiento.

El tiempo total del estudio fue de 12 meses, entre diciembre 2011 y diciembre 2012. En la figura 1 se detallan gráficamente los distintos tiempos del estudio y las variables registradas en cada momento. Se recogieron datos demográficos y clínicos (diagnóstico, tratamientos previos, episodios previos relacionados relevantes) al inicio del estudio. La medición del dolor se realizó mediante escala visual analógica (EVA de 0-10, $10 \mathrm{~cm}$, donde 0 indica ausencia de dolor, mientras que 10 indica dolor intratable) (Fig. 2). Para la evaluación de la función se aplicó el cuestionario de Ontario McMaster Universidades Western (WOMAC); se basa en escalas Likert que permiten que el paciente autoevalúe su estado. La puntuación WOMAC tiene tres subapartados: dolor (5 preguntas, 0-20), rigidez (2 preguntas, 0-8), y el funcionamiento físico (17 preguntas, 0-68), y tiene una puntuación mínima de 0 (mejor puntuación) y una puntuación máxima de 96 puntos (la peor) (9).

La respuesta al tratamiento se definió como $\geq 50 \%$ de disminución de la EVA del dolor (10). El grado de satisfacción del paciente se registró a los 6 meses después del

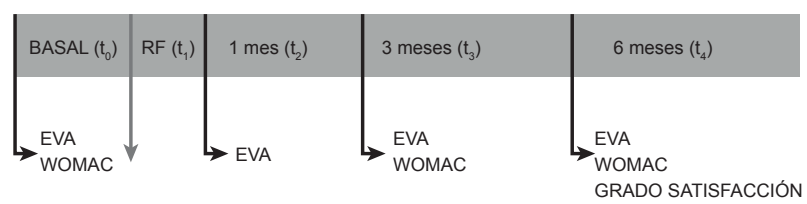

Fig. 1. Esquema temporal del estudio.

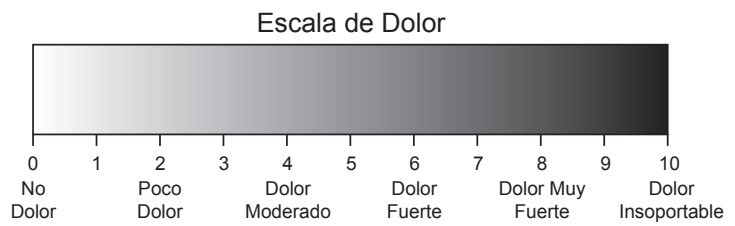

Fig. 2. Escala visual analógica. tratamiento: muy satisfecho, satisfecho, moderadamente satisfecho y nada satisfecho.

También se valoró la reducción en la medicación del paciente antes y después de la radiofrecuencia convencional.

Durante el seguimiento de estudio se registraron la aparición de episodios dolorosos, su duración y el tratamiento recibido, así como los efectos adversos o complicaciones debidos al procedimiento.

\section{Procedimiento}

La técnica se realizó en quirófano en condiciones idénticas de asepsia, con monitorización básica (pulsioximetría, ECG, TA) y guiados por fluoroscopia y neuroestimulación sensitivo-motora. Participaron 3 facultativos especialistas en anestesiología y formación en las técnicas de radiofrecuencia para el tratamiento del dolor, que forman parte de la Unidad de Dolor Crónico del Área Sanitaria de Jerez.

Anatómicamente los nervios geniculados de la rodilla derivan en la mayoría de los casos de los nervios tibial y peroneo (división a nivel de hueco poplíteo del nervio ciático). El nervio tibial proyecta ramas articulares a nivel de la fosa poplítea. Estas ramas articulares son la SM, medio e IM nervios geniculares. El nervio peroneo común también provee de ramas articulares, especialmente las SL, IL, y recurrente del nervio tibial geniculado. Las SL, SM e IM nervios que acompañan a los vasos geniculados pasan cerca de epicóndilo del fémur y la tibia, a excepción del nervio genicular IL, que se extiende lateralmente por encima de la cabeza del peroné, y no pasa cerca del epicóndilo lateral de la tibia (8).

El paciente se coloca en la mesa de quirófano en decúbito supino con la pierna en ligera flexión $\left(20^{\circ}\right)$. Se localizan los nervios geniculados de la cápsula articular de la rodilla: el lateral superior (SL), superior medial (SM), inferior lateral (IL), que pasan por el área perióstica de conexión del eje del fémur a epicóndilos bilaterales y al eje de la tibia para el epicóndilo medial (Fig. 3).

De forma percutánea tras la infiltración de anestésico local (mepivacaína $2 \%$ ) en cada punto, se insertan unas agujas de radiofrecuencia (Cosman $®$ electrode CR-P10 de $10 \mathrm{~cm}$ de longitud y $23 \mathrm{G}$ de ancho, con punta activa de $5 \mathrm{~mm}$ ), en visión tunel hasta alcanzar, los nervios geniculados mediante fluoroscopia, por referencias anatómicas anteriormente descritas. En este caso se emplea estimulación sensitiva $(50 \mathrm{~Hz})$ con $0,5 \mathrm{~V}$ (parestesia concordante con el dolor) y motora $(2 \mathrm{~Hz})$ a $1,2 \mathrm{~V}$ (no debe existir respuesta motora), la impedancia se sitúa entre 300-700 $\Omega$. Una vez localizadas las tres dianas, se realiza el tratamiento de radiofrecuencia a través del generador (Cosman ${ }^{\circledR}$ RFG-1a Generator Cosman Medical, Inc. Burlington, MA. USA). Se emplea el modo convencional durante 90 segundos a $20 \mathrm{~V}$.

La administración la realiza personal muy experimentado y con controles de constantes, bajo supervisión médica per- 
manente, por lo que los riesgos son escasos. Se han observado algunos casos de dolor leve en la zona o hematoma leve que son transitorios y rara vez obligan a tomar medidas especiales. De manera esporádica, pueden notarse parestesias en la zona.

\section{Análisis estadístico}

Para el análisis estadístico de los datos se ha utilizado el software SPSS versión 16.0 (SPSS Inc, Chicago, IL. USA). Se ha estudiado la normalidad de las variables cuantitativas mediante el test de Shapiro.

Para variables cuantitativas, los resultados descriptivos se han expresado mediante medidas de tendencia central y de dispersión (media y desviación estándar). Las variables cualitativas se han expresado mediante número y porcentaje. Se ha realizado una comparación de medias de las EVA y de las medias resultantes de la escala WOMAC y SF-12 al inicio, al mes del procedimiento, a los 6 meses. Esta comparación de medias en los distintos tiempos registrados en el estudio se realizó mediante el test de Friedman no paramétrico. El grado de satisfacción se expresa en porcentaje. Se considera un nivel de significación estadística del $95 \%(\mathrm{p}<0,05)$.

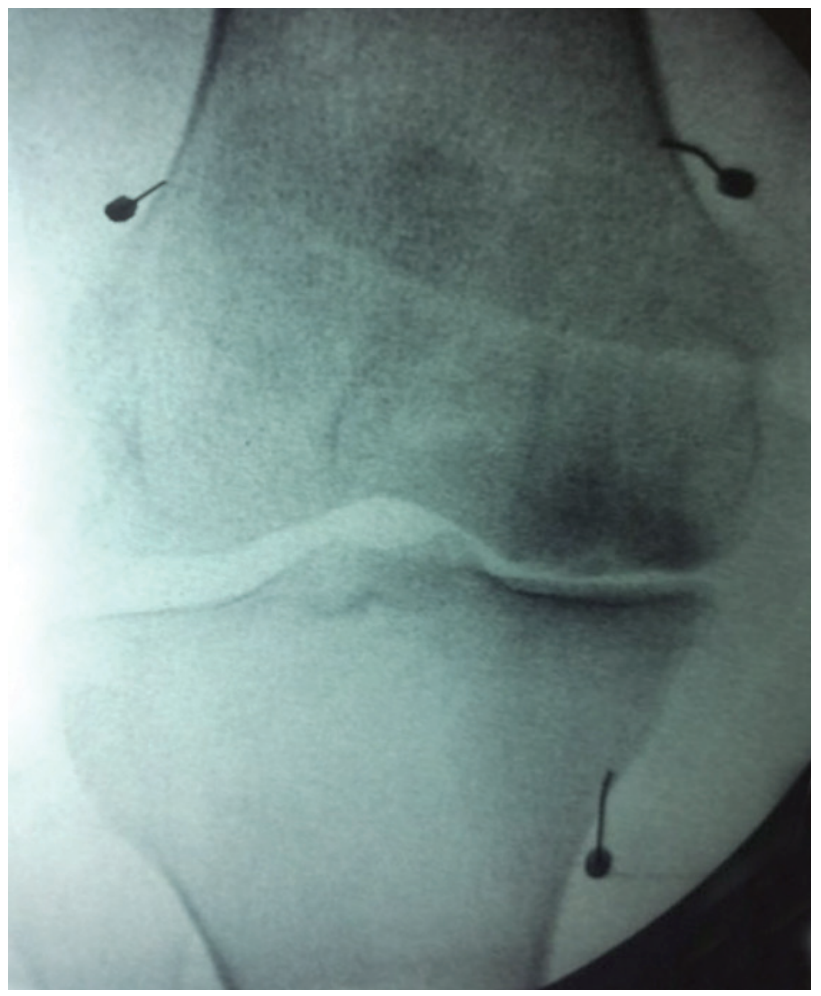

Fig. 3. Imagen radiológica puntos diana de radiofrecuencia: el nervio geniculado lateral superior (SL), superior medial (SM) e inferior medial (IL), que pasan por el área perióstica de conexión del eje del fémur a epicóndilos bilaterales y al eje de la tibia para el epicóndilo medial.

\section{RESULTADOS}

Se realiza el estudio observacional prospectivo de una muestra de 16 pacientes, en los que se realiza radiofrecuencia convencional de los nervios geniculados durante diciembre 2011 a diciembre 2012 en nuestra Unidad de Dolor Crónico. Con respecto a las variables demográficas, la edad media de los pacientes en los que se realizó radiofrecuencia fue de 74,5 años, con ASA medio de 2,8. La rodilla más afectada fue la derecha en el 68,75\% de los casos y el tiempo medio de evolución hasta ser estudiados en la Unidad del Dolor de 52 meses $( \pm 7,2)$ (Tabla I).

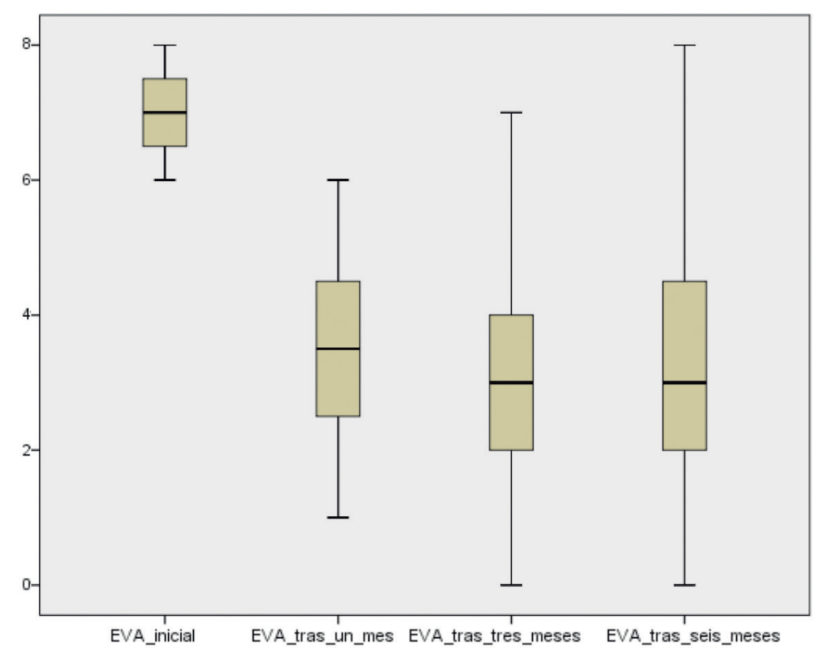

Fig. 4. Evolución de las puntuaciones en EVA en los meses de estudio. Diferencias significativas entre puntuación inicial y al mes, tres y seis meses. Test de Friedman (p:0,009).

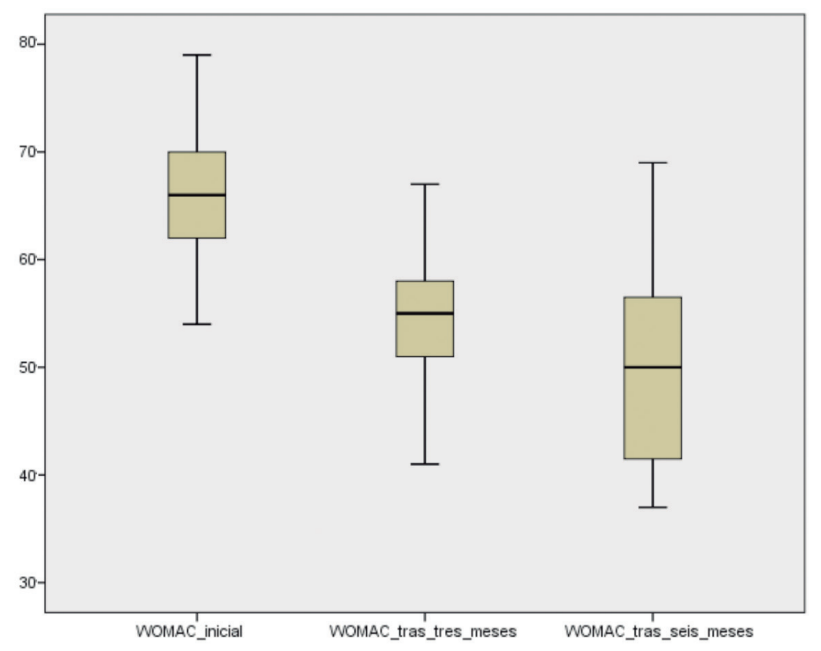

Fig. 5. Evolución de las puntuaciones en escala WOMAC en los meses de estudio. Diferencias significativas entre puntuación inicial y a los tres y seis meses. Test de Friedman (p:0,01). 


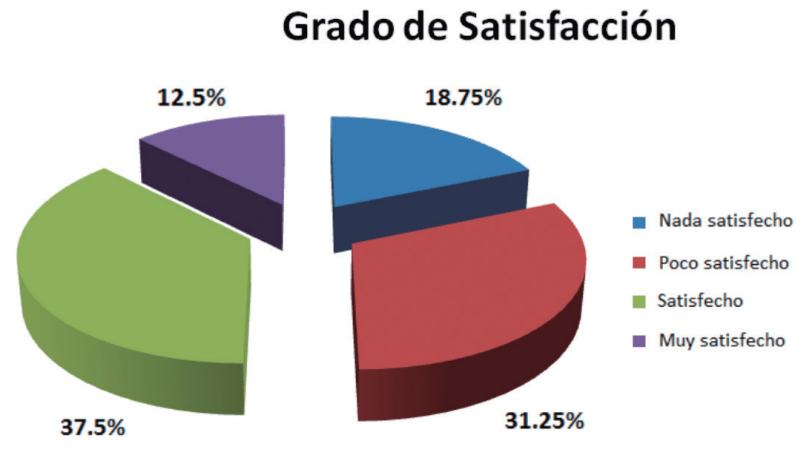

Fig. 6. Grado de satisfacción de los pacientes al final del tratamiento.

Con respecto al dolor medido mediante EVA obtuvimos una media inicial de 7 (6-8), 3,5 (1-6) al primer mes, 3,3 (1-7) a los tres meses y 3,37 (1-8) a los seis meses. Tras comparar las medias de EVA podemos afirmar que existen diferencias estadísticamente significativas entre el valor inicial y el valor al mes, tres y seis meses $(\mathrm{p}=0.009)$. El $75 \%$ de los pacientes presentó una reducción del EVA $\geq 50 \%$ en el primer mes, el $68,7 \%$ a los 3 meses, y el $65 \%$ a los 6 meses de tratamiento.

Con respecto a la mejoría del dolor y capacidad funcional medida mediante la escala WOMAC, se obtuvo una media inicial de 65,8 (54-79), de 54,7 (41-67) a los tres meses y 50,3 (37-69) a los seis meses. Tras comparar las medias de la puntuación de la escala WOMAC en los distintos momentos de medida, se encontraron diferencias estadísticamente significativas en cuanto a mejoría del dolor y aumento de la capacidad funcional entre la medición inicial y la medición a los tres y seis meses $(\mathrm{p}=0,01)$.

Al finalizar el tratamiento, el 18,7 \% de los pacientes se consideró nada satisfecho, el $31,25 \%$ poco satisfecho, el $37,5 \%$ satisfecho y el $12,5 \%$ muy satisfecho.
En ningún caso hubo un empeoramiento de la sintomatología de los pacientes. Únicamente una paciente no obtuvo beneficio alguno de la técnica desde el inicio del tratamiento. Dos pacientes refierieron zona hipoestésica por cara anterior de la rodilla tratada durante 3 meses.

El tratamiento médico se pudo reducir en 8 pacientes; de los cuatro que estaban el tercer escalón de la escalera analgésica de la OMS, tres pasaron al segundo escalón, y uno de ellos al primero.

No se objetivaron complicaciones derivadas de la técnica, realizándose la técnica en régimen ambulatorio, sin necesidad de ingreso en ningún caso.

\section{DISCUSIÓN}

$\mathrm{La}$ artrosis de rodilla sigue siendo una de las patologías más comunes en las unidades de dolor crónico, con una prevalencia alta en personas de edad avanzada.

Su tratamiento continúa siendo un reto. Existen diferentes opciones terapéuticas.

En el tratamiento habitual de la gonartrosis moderadasevera se recomienda la disminución de peso corporal para reducir el daño articular, los programas de ejercicio físico isométricos de fortalecimiento, la termoterapia con frío y calor, el uso de instrumentación de apoyo o contención articular, el tratamiento farmacológico (paracetamol o AINE, y como segunda línea inhibidores Cox 2 selectivos) y la estimulación transcutánea eléctrica del nervio. En general estos tratamientos no son suficientes en pacientes en estadios avanzados. Además el uso continuado de antiinflamatorios está asociado a serios efectos adversos, tales como lesión gastroduodenal, sangrados, alteraciones vasculares, disminución de la filtración glomerular a nivel renal $(5,6)$.

En numerosas ocasiones los pacientes son remitidos a la unidad de dolor por fracaso del tratamiento farmacológico de

TABLA I. DATOS SOCIODEMOGRÁFICOS. DATOS EXPRESADOS EN MEDIA Y SUS DESVIACIONES ESTÁNDAR

\begin{tabular}{lc} 
Número de pacientes & 16 \\
Edad (años) & $74 \pm 2$ \\
Sexo (porcentaje hombre/mujer) & $56 / 44$ \\
IMC & $28,3 \pm 1,9$ \\
ASA & $2,8 \pm 0,21$ \\
Duración síntomas dolorosos (meses) & $52 \pm 7,2$ \\
Tiempo en lista de espera (meses) & $3,2 \pm 2,4$ \\
Gradación Kellgren de artrosis en porcentaje (I, II, III, IV) & $0 / 0 / 56,25 / 43,75$ \\
Cirugía previa (\%) & 31,25 \\
Rodilla tratada (derecha/izquierda) & $68,75 / 31,25$ \\
Reducción dolor mayor del 50 \% tras tratamiento a los 6 meses (\%) & 62,5 \\
Descenso de al menos un escalón analgésico (\%) & 37,5 \\
Pacientes desestimados de cirugía (\%) & 6,25 \\
\hline
\end{tabular}


primera línea. En estos casos conjuntamente con analgésicos orales, se suelen utilizar opioides débiles, tramadol e incluso analgésicos narcóticos, combinados o no con la aplicación intraarticular de corticoides o el empleo de viscosuplementación intraarticular (11). Finalmente, el desbridamiento artroscópico, la osteotomía tibial alta y la cirugía de reemplazo articular constituyen las últimas opciones de tratamiento a decidir entre paciente y cirujano. La cirugía de reemplazo articular reduce el dolor y consecuentemente la incapacidad física y se considera un instrumento de mejora de la calidad de vida, aunque existe una proporción significativa de pacientes en los que persiste dolor crónico, la discapacidad, y una mala calidad de vida o insatisfacción con los resultados de esta (12). En estos pacientes con mala respuesta a la cirugía, en aquellos en los que esta se desestima por la existencia de comorbilidad asociada (obesidad mórbida o cardiopatía severa), o durante el tiempo de espera para la intervención (en ocasiones superior a los 6 meses), la radiofrecuencia puede ser una alternativa a emplear.

La técnica fue descrita por Choi y requiere la identificación de puntos de referencia anatómicos de los nervios que inervan la articulación de la rodilla, consiste en colocar la aguja, bajo visión radiológica y estimulación sensitivomotora, cercanos al periostio a nivel de los nervios geniculados superomedial (SM), superolateral (SL) e inferomedial (IL) y el depósito de la medicación en la proximidad de los mismos. La ventaja de esta técnica frente a las dos anteriores es que permite depositar la medicación en los puntos deseados, donde conectan el eje del fémur con epicóndilos bilaterales y el eje de la tibia con el epicóndilo medial, y colocar correctamente el electrodo paralelo al nervio diana, siguiendo el principio fundamental de RF (8).

En este pequeño ensayo clínico realizado por Choi y cols. encontraron un alivio significativo del dolor y una mejoría de la funcionalidad aplicando radiofrecuencia convencional sobre los nervios geniculados en una serie de pacientes ancianos, con pocos efectos adversos.

Nuestros resultados en la evolución de la EVA son semejantes a los obtenidos en el ensayo clínico realizado por Choi, encontrando un alivio significativo del dolor en los 3 primeros meses $(68,75 \%$ vs. $59 \%)$, considerando como se hace en estos trabajos que el dolor se disminuya al menos el $50 \%$. En nuestro estudio hicimos un seguimiento a los 6 meses, manteniédose un alivio significativo del dolor, con respecto al EVA basal. Esta mejoría se mantuvo en un $62,5 \%$ de los pacientes al final del estudio.

En nuestra serie la mejoría global de los pacientes estuvo por encima del $50 \%$ en todos los periodos del estudio; únicamente una paciente $(6,25 \%)$ no obtuvo mejoría alguna. Se trataba de una mujer de 71 años con un tiempo de evolución de 120 meses, pluripatológica en la que se había desestimado la cirugía.

Aunque los nervios geniculados son las principales ramas articulares de la rodilla, también llegan ramas arti- culares del nervio femoral, peroneo, los nervios safeno, tibial y del obturador. Por esta razón, pueden existir casos en los que el dolor de la articulación de la rodilla no puede ser completamente aliviado, lo que resulta en una mala respuesta a la RF. Debido al escaso número de pacientes es difícil poder sacar conclusiones al respecto.

En nuestra serie no tuvimos ninguna complicación con la aplicación del tratamiento, al contrario de lo publicado en el estudio de Choi, que informó de un paciente que sufrió una caída tres semanas después del tratamiento y otro paciente que desarrolló un hemartros. Únicamente dos pacientes refirieron pequeños hematomas en el sitio de punción.

En un comentario realizado por Kvarstein sobre estos efectos, este autor refiere que no fueron necesariamente relacionados con la técnica pero que podrían reflejar un deterioro en la estabilidad de la articulación después de la ablación nerviosa, incluso aunque los test clínicos no demuestren debilidad muscular, parestesias o propiocepción anormal. Es importante recordar que la RF convencional es una técnica destructiva con una coagulación no selectiva de fibras sensoriales (mielínicas, amielínicas), así como también de fibras motoras. De hecho, datos experimentales en perros sugieren que la propiocepción es importante para proteger la estabilidad de la articulación. Esta lesión se produciría porque la lesión por radiofrecuencia no discrimina entre fibras sensoriales, y porque hay que tener en cuenta que una respuesta negativa al estímulo motor no garantiza la posible lesión de fibras motoras (13).

No hemos encontrado en la literatura ningún otro estudio que haga referencia a la utilización de la radiofrecuencia en el tratamiento de la gonartrosis a nivel de nervios geniculados. Sí los hay de radiofrecuencia pulsada intraarticular, radiofrecuencia pulsada del nervio safeno $(14,15)$. Este último estudio retrospectivo de 115 pacientes presenta, tras la aplicación de radiofrecuencia pulsada sobre el safeno (40 V, $8 \mathrm{~min}$ ), buenos resultados, mejorando el $84,33 \%$ de los pacientes en cuanto a reducción del dolor medido mediante escala EVA y funcionalidad mediante cuestionario WOMAC grado de satisfacción de paciente más allá de los 6 meses. Podría ser interesante (15).

Ikeuchi y cols. desarrollaron un estudio prospectivo en 2011 en 35 pacientes, anterior al de Choi, en el que aplicaban RF convencional durante 90 segundos a nivel de la rama infrapatelar del safeno y nervio reticular medio (rama terminal del nervio femoral), mediante referencia anatómica ósea, con neuroestimulación sensitiva, sin control radioscópico. Los resultados fueron breves en el tiempo. El $50 \%$ de los pacientes obtuvo una reducción de $>50 \%$ del EVA y WOMAC en el primer mes, reduciéndose a los 6 meses ese porcentaje a menso del $10 \%$ de los pacientes. Todos los pacientes presentaron hipoestesia prolongada en el área (16). La ausencia de control radiográfico parece dificultar el procedimiento, de hecho este autor describe gran dificultad a la hora de posicionar la aguja a nivel de 
Rev. Soc. Esp. del Dolor, Vol. 21, N. ${ }^{\circ}$ 4, Julio-Agosto 2014

las estructuras diana. Aún no hay estudios suficientes que aporten pruebas fiables, efectivas y válidas sobre la localización de las estructuras nerviosas y las referencias anatómicas que sirvan de diana.

Otro estudio retrospectivo de 31 pacientes evaluó la eficacia de la RFP intraarticular $\left(15 \mathrm{~min}, 42^{\circ}\right)$ objetivando que sólo el $35,5 \%$ de los pacientes presentaba un alivio del dolor $(\mathrm{EVA})>50 \%$ a los 6 meses del tratamiento (14).

No parece lo más adecuado desde el punto de vista metodológico, comparar los resultados alcanzados de radiofrecuencia convencional a nivel de geniculados con los obtenidos con radiofrecuencia pulsada a nivel intraarticular y del safeno, pero podría ser interesante evaluar la eficacia de la radiofrecuencia combinada de geniculados y safeno.

En este trabajo preliminar presentamos nuestros resultados en 16 pacientes que presentaban gonalgia rebelde a otros tipos de tratamiento. Las conclusiones que podemos sacar del estudio son limitadas por el escaso número de pacientes incluidos, así como por la falta de aleatorización y de un grupo control con el que podamos comparar el tratamiento efectuado en nuestros pacientes. De hecho, actualmente estamos llevando a cabo un estudio prospectivo con una muestra mayor, para evaluar la eficacia de la RF pulsada de los nervios geniculados en pacientes con gonartrosis, de este modo, evitaríamos teóricamente la lesión de tejidos y fibras que pudieran estar en relación con la estabilidad de la articulación y la propiocepción.

\section{CONCLUSIONES}

En conclusión, la RF convencional parece reducir de manera significativa la intensidad del dolor en un periodo de 6 meses en más del $50 \%$ de los pacientes, al igual que mejora la capacidad funcional en pacientes diagnosticados de osteoartrosis de rodilla. Son necesarios estudios más amplios, comparativos y prospectivos para poder realizar la evaluación de la eficacia de esta técnica.

\author{
CORRESPONDENCIA: \\ Ivan Ramírez \\ Servicio de Anestesia \\ Hospital de Jerez de la Frontera \\ Ronda de Circunvalación, s/n \\ 11407 Jerez de la Frontera - Cádiz \\ e-mail: ivan_ramirez_ogalla@hotmail.com
}

\section{BIBLIOGRAFÍA}

1. Garstang SV, Stitik TP. Osteoarthritis epidemiology, risk factors and pathophysiology. Am J Phys Med Rehabil 2006;85(11 Supl.):S2-11.
2. Carmona L, Gabriel R, Ballina F, Laffon AY. Proyecto EPISER 2000: Prevalencia de enfermedades reumáticas en la población española. Metodología, resultados del reclutamiento y características de la población. Rev Esp Reumatol 2001;28(1):18-25.

3. Loza E, Jover JA, Rodriguez L, Carmona L; EPISER Study Group. Multimorbidity: Prevalence, effect o quality of life and daily, functioning, and variation of this effect when one condition is a rheumatic disease. Semin Arthritis Rheum 2009;38(4):312-9.

4. Conaghan PG, Dickson J, Grant RL, Guideline development group. Guidelines: Care and management of osteoarthritis in adults: Summary of NICE guidance. BMJ 2008;336(7642):502-3.

5. Zhang W, Nuki G, Moskowitz RW, Abramso S, Altman $\mathrm{RD}$, Arden NK, et al. OARSI recommendations for the management of hip and knee osteoarthritis. Part III: Changes in evidence following systematic cumulative update of research published through January 2009. Osteoarthritis Cartilage 2010;18 (4):476-99.

6. Roddy ED, Doherty M. Guidelines for management of osteoarthritis published by the American College of Rheumatology and the European League against Rhumatism: Why are they so different? Rheum Dis Clin North Am 2003;29:717-31.

7. Raj PP, Lou L, Erdine S, Staats PS, Waldman SD, Racz $\mathrm{G}$, et al. Interventional pain management: Image-guided procedures. 2nd ed. Philadelphia: Saunders Elsevier; 2008.

8. Choi WJ, Hwang SJ, Song JG, Leem JG, Kang YU, Park $\mathrm{PH}$, et al. Radiofrequency treatment relieves chronic knee osteoarthritis pain: A double-blind randomized controlled trial. Pain 2011;152:481-7.

9. Escobar A, Quintana JM, Bilbao A, et al. Validation of the Spanish version of the WOMAC questionnaire for patients with hip or knee osteoarthritis. Western Ontario and McMaster Universities Osteoarthritis Index. Clin Rheumatol 2002;21:466-71.

10. Pham T, van der Heijde D, Altman RD, Anderson JJ, Bellamy N, Hochberg M, et al. OMERACT-OARSI initiative: Osteoarthritis Research Society International set of responder criteria for osteoarthritis clinical trials revisited. Osteoarthritis Cartilage 2004;12:389-99.

11. Hochberg MC, Altman RD, April KT, Benkhalti M, Guyatt G, McGowan J, et al. American College of Rheumatology 2012 recommendations for the use of nonpharmacologic and pharmacologic therapies in osteoarthritis of the hand, hip, and knee. Arthritis Care \& Research 2012;64(4):465-74.

12. National Collaborating Centre for Chronic Conditions. Osteoarthritis: National clinical guideline for care and management in adults. London: Royal College of Physicians, 2008. Available at: http://www.nice.org.uk/nicemedia/live/11926/39557/39557.pdf

13. Kvarstein G. A new target for radiofrequency neurotomy? Pain 2011;152(3):465-6.

14. Karaman H, Tüfek A, Kavak GÖ, Yildirim ZB, Uysal E, Celik F, et al. Intra-articulaly applied pulsed radiofrequency can reduce chronic knee pain in patients with osteoartritis. J Chin Med Assoc 2011;74(8):336-40.

15. Akbas M, luleci N, Dere K, Ozdemir U, Toman H. Efficacy of pulsed radiofrequency treatment on the saphenous nerve in patients with chronic knee pain. J Back Musculoskelet Rehabil 2011;24(2):77-82.

16. Ikeuchi M, Ushida Takahiro, Masashi I., Toshikazu T. Percutaneous radiofrequency treatment for refractory anteromedial pain of osteoarthritic knees. Pain Medicine 2011;12:546-51. 